
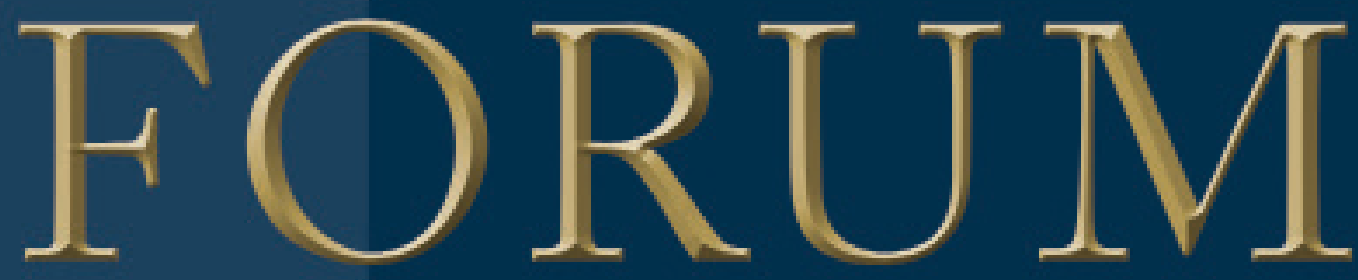

VOLUME 28 I NUMBER 3 MAY/JUNE

HAIR TRANSPLANT FORUM INTERNATIONAL

IN THIS ISSUE

Hair Restoration in Cranial Surgery Patients

A New Multi-Graft Implanter System

$\overline{\text { MRSA }}$ and Hair: Then and Now

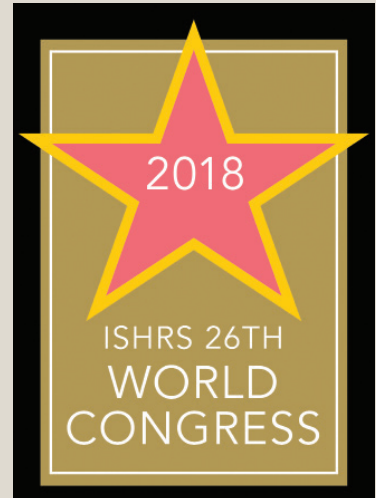

OCTOBER 10-14 HOLLYWOOD LOEWS HOLLYWOOD HOTEL www.ISHRS.org

\section{A Histological and Clinical Evaluation of Plasma as a Graft Holding Solution and Its Efficacy in Terms of Hair Growth and Graft Survival}

Anil Kumar Garg, MBBS, MS.MCh, FISHRS I Indore, India I anilgarg61@yahoo.com; Seema Garg, MBBS, MSc I Indore, India

Disclosure: Authors have no conflict of interest.

\section{INTRODUCTION}

The surgical trauma of hair transplantation triggers inflammation, which is the first step in wound healing. The brunt of the biochemical changes has to be borne by the newly transferred grafts, which are devoid of any blood supply. Graft survival is affected by a multitude of factors including graft harvesting, dissection, manipulation during implantation, and ischemia/reperfusion injury following implantation in the body. The insults inflicted from these unfavourable factors lead to apoptosis, which affects graft survival and the quality of hair regrowth. In order to achieve the best results, we should focus on improving all of the above mentioned factors.

Grafts harvested and maintained out of the scalp are preserved in a holding solution until they are implanted. Thus, the holding solution plays a crucial role in the hair transplant procedure. An ideal holding solution should have the same osmolality as the graft cells, should prevent acidosis, should provide energy to the cells, and should prevent the release of free radicals. There are two types of holding solutions: extracellular and intracellular. Examples of extracellular solutions are normal saline, Lactated Ringer's (LR), and plasmalike fluids. The intracellular solutions are represented by HypoThermoso ${ }^{\circledR}$. Extracellular holding solutions do not require chilling, which causes sodium pump failure leading to swelling of the cells, whereas intracellular holding solutions do require chilling.'

At our center, we use autologous plasma with platelets as a graft holding solution during hair transplantation surgery. Clinical results have been evaluated with trichoscan analysis and supported by histological evaluation for graft viability. There is literature advocating the use of platelet-rich plasma (PRP) to promote hair growth, based on the logic that platelets have growth factors that stimulate the stem cells of hair follicles. ${ }^{2}$

\section{OBJECTIVE}

To evaluate plasma as a graft holding solution in terms of its efficacy in hair growth and hair graft survival.

\section{METHOD}

A split-scalp study was carried out comparing grafts transplanted on the right and left fronto-temporal areas selected as recipient sites in the same patient. In the initial study, we used mainly FUT grafts, and in the last phase, we used FUE grafts. A total of 6 patients have been enrolled in the study so far. The left side was designated as the control area (Group A), and the right side behaved as the test area (Group B), for comparison of the results. The right fronto-temporal area received grafts preserved in autologous plasma, while the left fronto-temporal area received grafts preserved in LR solution. Both sides were implanted with grafts harvested using the same technique, with an equal number of grafts of the same quality, and with the same implantation time. Grafts on both sides were implanted by two surgeons sharing similar experience and expertise using optical loupes for magnification (4.5x).

Both the autologous plasma and the LR holding solutions with the grafts were maintained at a temperature of approximately $12 \pm 2^{\circ} \mathrm{Celsius,}$ whereas the room temperature was maintained around $18^{\circ} \mathrm{Celsius}$. 
The following parameters were taken into consideration for the study:

- A histological study with MTT stain (a colorimetric assay for assessing cell metabolic activity) was done in order to confirm the viability of cells in the grafts at 12 hours and 72 hours.

- Periodical post-operative patient follow-up with regular photographs and trichoscan evaluations was used to identify any event of anagen effluvium due to post-surgical shock loss.

- Trichoscan study for hair density was done at 3 months for hair growth.

- Hair thickness was assessed at 6 months and 12 months for the quality of hair growth.

\section{Preparation of autologous plasma}

Preparation of autologous plasma was the first step before performing the hair transplant. We collected 23cc of blood from the patient in a syringe with 2cc ACD (acid citrate dextrose) solution as an anti-coagulant. The blood was transferred to a high-quality glass container designed by the author. The blood was centrifuged in a temperature-controlled $\left(19^{\circ}\right.$ Celsius) centrifuge machine at 5,000 RPM (rotations per minute) for 16 minutes. The process resulted in the separation of red blood cells (RBCs) at the bottom of the tube and plasma with platelets forming the upper fluid compartment. The 23cc of blood yielded approximately $12 \mathrm{cc}$ of plasma. Hence, we can deduce that the platelet concentration was twice normal levels. The lab further confirmed that the platelet count ranged between $400,000-500,000 / \mathrm{mm}^{3}$. The plasma created was then stored in a sterile stainless steel bowl (a petri dish can also be used) maintaining a temperature of $12^{\circ} \pm 2^{\circ}$ Celsius on a cool gel pack ready to receive the grafts.

The harvested grafts were divided randomly into two groups, with an equal number of grafts per side. Control Group A grafts were stored in LR solution and test Group $B$ were stored in plasma solution. The temperature of both graft holding solutions was maintained as the same. Grafts dipped in plasma formed a very loose clump. A trained assistant separated the individual grafts from a small clump and placed it on the surgeon's hand to implant.

Six volunteer patients ranging in age between 25 to 40 years old and having similar grades of male pattern hair loss $(\mathrm{MPHL})$ were included in the study. Grafts were implanted over the bilateral fronto-temporal areas as planned. Grafts stored in plasma solution were implanted on the right fronto-temporal side and the grafts stored in LR solution were implanted on the left fronto-temporal side. The same number of grafts were implanted resulting in a standard density of 40 grafts $/ \mathrm{cm}^{2}$. Routine post-operative care of the donor and the recipient areas was followed as per general guidelines for all hair transplant patients. The study was conducted as follows:

- The graft samples A and B were sent for MTT staining at 12 hours and 72 hours of graft holding time in order to determine viability of the cells.

- Patient follow-ups were conducted at 1, 2, 3, 4, 6, and 12 months after transplant.

- Photographs were taken for comparison of the left and right fronto-temporal areas with and without flash.

- Hair count and density were taken on both sides using trichoscan.

- At the 6- and 12-month follow-ups, photographs and trichoscan for hair thickness were repeated for evaluation of terminal hair.

\section{OBSERVATION}

MTT staining at 12 hours showed that grafts stored in LR solution showed poor staining, while the grafts stored in plasma solution were well stained, indicating good cell viability in the plasma group when compared to the LR group

(Figure 1). MTT

staining at 72 hours showed grafts stored in plasma showed good staining while the LR group showed very poor staining.

\section{Trichoscan study}

As shown in Table 1, the hair count and density in the plasma group were significantly higher than in the LR group. The unpaired t-test showed the LR group had a mean $4.5 \pm$ 2.95 SD and the plasma group had mean 27.50 \pm 4.135 SD with P-value $<.001$.

TABLE 1. Hair Count and Density at 3 Months on LR Side and Plasma Side (Implanted density: 40 grafts/ $\mathrm{cm}^{2}$ )

\begin{tabular}{|c|c|c|c|c|c|c|}
\hline \multirow[b]{2}{*}{ 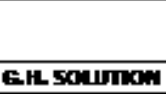 } & \multicolumn{2}{|c|}{ Hir cout } & \multicolumn{2}{|c|}{ 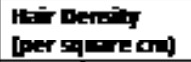 } & \multicolumn{2}{|c|}{ 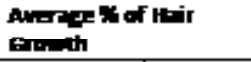 } \\
\hline & n & PLATIA & मL & Fucth & pl & Facis \\
\hline & $\mathbf{Z}$ & 9 & E. & 20.7 & \multirow{7}{*}{$1101 x$} & \multirow{7}{*}{ EgTK } \\
\hline & 1 & in & $\mathbf{3 3}$ & 코 & & \\
\hline & 2 & 7 & ES & 23.1 & & \\
\hline & 0 & E & no & 25.4 & & \\
\hline & $\mathbf{z}$ & 7 & EБ & 23.1 & & \\
\hline & 1 & 9 & $\mathbf{3 3}$ & 2.7 & & \\
\hline AVP:16E & 13 & 53 & 44 & 25 & & \\
\hline
\end{tabular}

\section{CLINICAL EVALUATION}

Photographs of the right and left fronto-temporal areas were taken at 50 days (Figure 3) and 120 days (Figure 4) after hair transplant for evaluation of hair growth and anagen effluvium. Also shown is a patient 120 days after transplant. Plasma holding solution was used for all grafts (Figure 5).

\section{DISCUSSION}

The most important benefit of a graft holding solution would be an increase in hair yield from the transplanted grafts. The optimum holding solution would reduce the damage from reperfusion injury and free radical formation as well as from ionic imbalance and variation in osmolality created by the ischemic phase.

Holding solutions are formulated according to the composition of intracellular and extracellular body fluid environment 
FIGURE 3. Immediate post-op (top) and 50 days post-op: plasma (bottom left), Lactated Ringer's (bottom right)
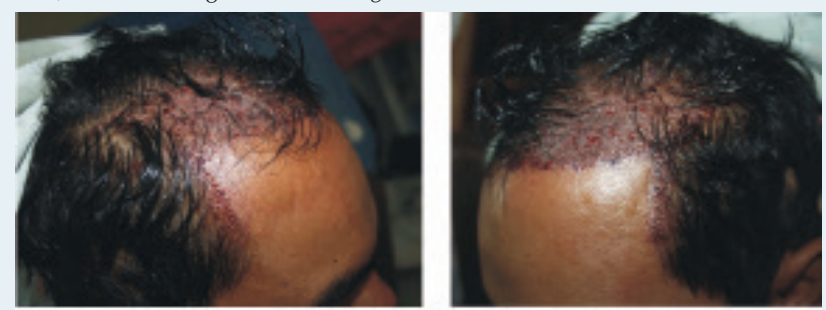

50 DAYS POST OP

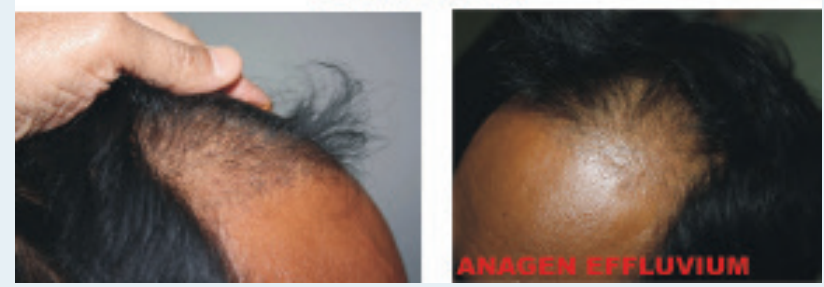

FIGURE 4. Pre-op (top) and 4 months post-op: patient's right hairline (bottom left)plasma, left hairline-LR; LR side (middle photo); plasma side (right photo)

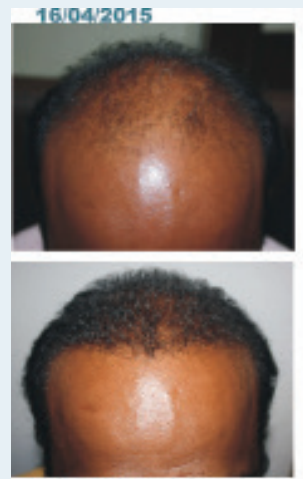

$14 / 08 / 2015$
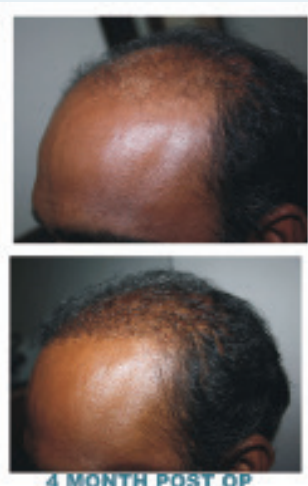

4 MONTH POST TP
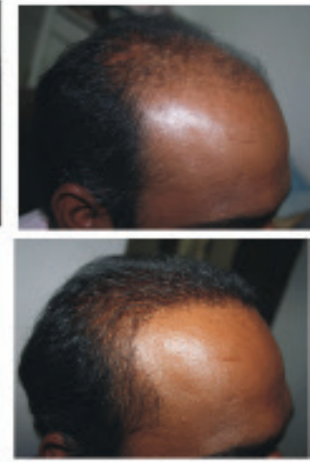

FIGURE 5. Pre-op (top) and 4 months post-op (bottom); both sides plasma holding solution

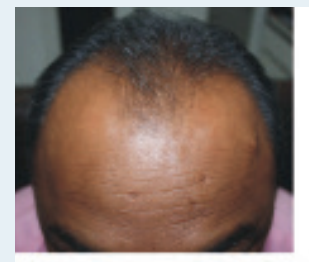

RESULT AT END OF 4 MONTHS
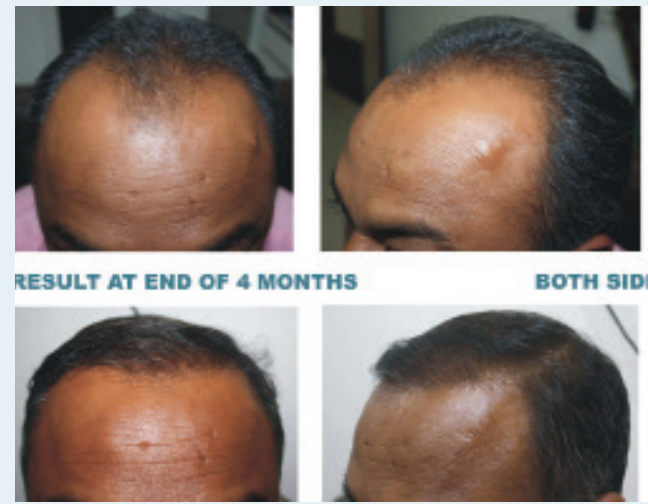

BOTH SIDE PLASMA
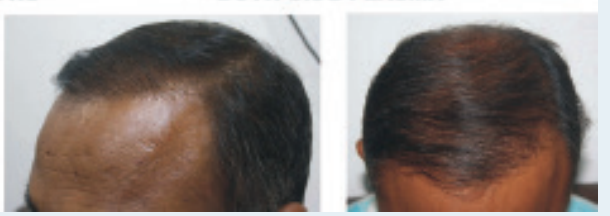

and behave differently. An intracellular graft holding solution needs chilling, which is not user-friendly. It also does not ensure protection from reperfusion injury and is expensive. ${ }^{3}$ Extracellular solutions are widely used, economical, and do not need chilling. Intracellular fluids like hypothermasol with ATP added have significant benefits when graft holding time is more than 10 hours, however, this is a very rare situation as most hair transplant procedures are complete within 4-6 hours.

Autologous plasma is an extracellular fluid that is isotonic with nutrients and platelet-derived growth factors. It is cost effective and can be prepared by a surgeon or a pathologist. Drying and dessication of grafts immersed in plasma is delayed, and grafts look shiny and more hydrated even at the end of 4 hours holding time. Ubel in his study had implanted grafts after dipping in plasma and reported a 5 to $53 \%$ increase in hair count after 7 months of hair transplant. ${ }^{2,6}$

\section{MTT (3-(4,5-Dimethylthiazol-2-Yl)-2,5-Diphenyltetrazolium Bromide) Assay}

The MTT assay is a colorimetric assay for assessing cell metabolic activity. $\mathrm{NAD}(\mathrm{P}) \mathrm{H}$-dependent cellular oxidoreductase enzymes reflect the number of viable cells present under defined conditions. These enzymes are capable of reducing the tetrazolium dye MTT 3-(4,5-dimethyl-thiazole2-yl-2,5-diphenyl tetrazolium bromide) to its insoluble form. ${ }^{9}$ Therefore, the dye can detect metabolically active live cells. In our study, samples of hair follicle grafts were sent for MTT histological assay in order to detect live cells. Results of staining showed that at 12 hours the grafts held in plasma solution were better stained than those held in LR solution. Staining results at 72 hours were surprising: plasma grafts showed good staining while LR grafts showed very poor staining indicating that the cells were viable in plasma grafts even at the end of 72 hours.

Trichoscan study done at 3 months for the hair count on the LR side showed an average of 1.3 (density $4.4 \mathrm{~g} / \mathrm{cm}^{2}$ ); on the plasma side, the average hair count was 8.3 (density $27.5 \mathrm{~g} / \mathrm{cm}^{2}$ ). Thus, the plasma side had $68.75 \%$ hair growth, while the LR side had only $11 \%$ growth. The unpaired t-test showed mean $4.5 \pm 2.95 \mathrm{SD}$ for the LR side and mean 27.50 $\pm 4.135 \mathrm{SD}$ for the plasma side with a P-value $<.001$, which is significant. This indicates that anagen effluvium (Figure 6) on the plasma side was $31.25 \%$ while on LR side the effluvium was $89.00 \%$. This shows that anagen effluvium was controlled by $58.75 \%$, which is significant (P-value $<.001$ ).

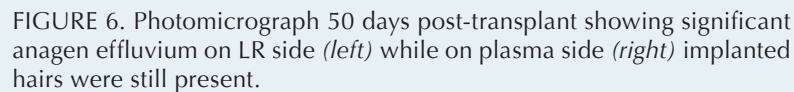
hairs were still present.
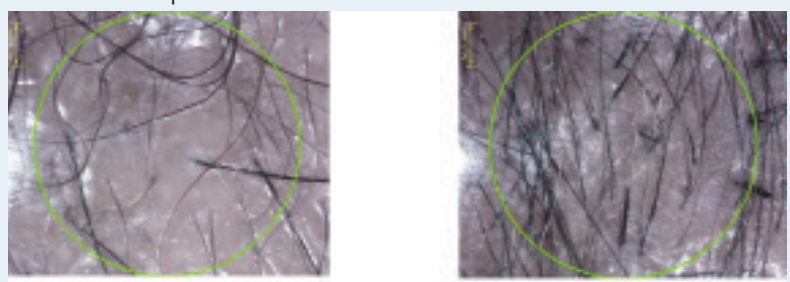

LACTATED RINGER'S ANAGEN EFFLUVIUM PLASMA
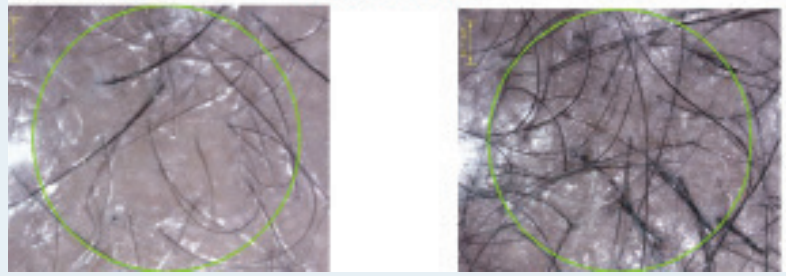

In the first 7 days following a hair transplant, there is a period of inflammatory response (involving neutrophils, eosinophils, macrophages, platelets, fibroblasts and growth factors ${ }^{2}$ ) in which both erythema and edema occur followed by apoptosis and the grafted, as well the existing, hair follicles may enter into an involution phase resulting in hair shedding. This process is triggered and propogated due to ischemia. The follicles become refractory and those that survive will regrow at the stimulus of 
the next growth cycle, which begins after the third month and continues up to the seventh month. Prevention of anagen effluvium can be achieved with prevention of apoptosis of the more metabolically active progeny of the stem cells. This observation may help us in the development of an ideal holding solution by further bio-enhancement of platelet and plasma solution.

Hair thickness measured at 6 months by trichoscan showed an average of $53.5 \mu \mathrm{mm}$ on the LR side while on plasma side it was $65.66 \mu \mathrm{mm}$, which was significantly higher (Figure 7). The unpaired t-test showed the LR group mean $53.5 \pm 6.377 \mathrm{SD}$ and the plasma group mean $65.67 \pm$ 9.688 SD. The P-value was <.001, which is very significant.

At the 12-month follow-up, hair thickness measured 60.6 $\mu \mathrm{mm}$ on the LR side while on the plasma side it was 66.125 $\mu \mathrm{mm}$. The thickness of hair on the LR side increased by 12 months but was still less than on the plasma side. The un-

FIGURE 7. Hair thickness in both groups at 6 months and 12 months was compared. There was an improvement in hair thickness in both groups from 6 months to 12 months with P-value .002.

HAIR THICKNESS AT 6 AND 12 MONTHS IN PLASMA AND LR

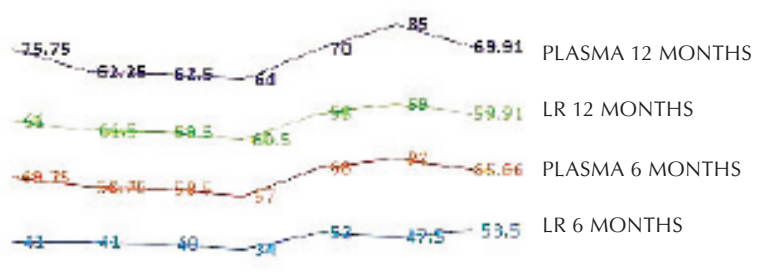

paired t-test showed the LR group mean $60.00 \pm 1.414 \mathrm{SD}$, and the plasma group mean $69.86 \pm 9.218$ SD. The P-value was $<.001$, which is very significant.

Hair diameter depends on a number of viable cells in the matrix. These are the mesodermal stem cells known to be very sensitive to ischemia. Ischemia leads to accumulation of free radicals and anaerobic metabolic pathways resulting in apoptosis of cells thereby affecting hair thickness. The hair thickness on the plasma graft side was better than on the LR side. This may be because of the effect of multiple beneficial factors in the plasma holding solution.

Platelets are activated on contact with collagen around hair follicles resulting in the release of various platelet derived growth factors. ${ }^{15,16}$ Fibrinogen in plasma gets converted to fibrin, which forms a mesh in which platelets are trapped. ${ }^{2,4-6}$ This fibrin mesh with activated platelets forms a 3D fibrin scaffold. Platelet-rich fibrin (PRF) was first described by Choukroun et al. in France. ${ }^{10,11}$ Fibrin glue along with skeletal myoblasts in the fibrin scaffold preserve cardiac function after myocardial infarction. ${ }^{12}$ In vitro prefabrication of human cartilage is created in shapes using fibrin glue and human chondrocytes. ${ }^{13}$ Long-term regeneration of human epidermis is achieved on third-degree burns transplanted with autologous cultured epithelium grown on a fibrin matrix. ${ }^{13}$ There is a definite role of the fibrin matrix in angiogenesis. ${ }^{14}$

\section{CONCLUSION}

Autologous plasma is an easily available graft holding solution. It is isotonic in nature having nutrient growth factors as well as the advantage of fibrin. Platelets along with the plasma provide multiple growth factors promoting epithelialization and neovascularization, and action on hair follicle stem cells to improve growth. The fibrin coating around the graft makes it sticky and prevents dehydration. The growth factors and nutrients successfully prevent the anagen effluvium and shock loss post hair transplant. The thickness of hair and yield of the graft is also better in plasma. The split-scalp controlled study certainly shows the advantages of using plasma over other extracellular graft holding solutions. At the same time, it is not an ideal graft holding solution where chilling cannot be done and the availability of energy source is not clear. But this can be developed as an ideal graft holding solution by some innovative bioenhancement.

\section{References}

1. Cole, J.P. (2012) Internet website posting. www.forhair.com/ optimal-holding-solution-and-temperature-for-hair-follicle/

2. Uebel, C.O. A new advance in baldness surgery using platelet-derived growth factor. Hair Transplant Forum Int'l. 2005; 15(3):77-84. (Micrografts stored in platelet rich plasma showed greater survival compared to those stored in saline.)

3. Cooley, J. Ischemia reperfusion injury and graft storage solutions. Hair Transplant Forum Int'l. 2004; 13(4):121.

4. Miao, Y., et al. Promotional effect of platelet rich plasma on hair follicle reconstitution in vivo. Dermatol Surg. 2013; 39:1868-1876.

5. Greco, J. Preliminary experience and extended applications for the use of autologous platelet rich plasma in hair transplantation surgery. Hair Transplant Forum Int'l. 2007; 17(4):131-132.

6. Uebel, C.O., et al. The role of platelet plasma growth factors in male pattern baldness surgery. Plast Recontr Surg. 2006; 118(6):1458-1466.

7. Deyarman Internet website posting. www.hairtransplantnetwork. com/Hair-Loss- Treatments/Platelet_Rich_Plasma_PRP_Hair_ Transplant.asp

8. Garg, A., and S. Garg. A histological and clinical evaluation of plasma as a graft holding solution and its efficacy in terms of hair growth and graft survival. Paper presented at the Annual World Congress of the ISHRS. Las Vegas, California, USA, 2016.

9. MTT assay. Wikipedia: https://en.wikipedia.org/wiki/MTT_assay.

10. Choukroun, J., et al. Platelet-rich fibrin (PRF): A second generation platelet concentrate: Part I: Technological concepts and evolution. Oral Surg Oral Med Oral Pathol Oral Radiol Endod. 2006; 101:E37-44.

11. Sunitha, R.V., and N. E. Munirathnam. Platelet-rich fibrin: Evolution of a second-generation platelet concentrate. Indian J Dent Res. [serial online] 2008 [cited 2017 Sep 11]; 19:42-46.

12. Karen, L., et al. Fibrin glue alone and skeletal myoblasts in a fibrin scaffold preserve cardiac function after myocardial infarction. Journal of Tissue Engineering. 2004(Mar); 10(3-4):403-409.

13. Ting, V., et al. In-vitro prefabrication of human cartilage shapes using fibrin glue and human chondrocytes. Annals of Plastic Surg. 1998; 40(4):413-421.

14. Van Hinsbergh, C.A., and P. Koolwijk. Role of fibrin matrix in angiogenesis. Ann N Y Acad Sci. 2001; 936:426-437.

15. Seong-Hoon, Y., et al. Platelet activation: the mechanisms and potential biomarkers. BioMed Research Int'l. Volume 2016 (2016). Article ID 9060143, 5 pages.

16. Platelet Activation. https://courses.washington.edu/conj/ bloodcells/platelets.htm 